\title{
Dual wavelength Talbot self-imaging using facet patterned nonlinear crystals
}

\author{
A. M. Vyunishev, ${ }^{1,2, *}$ (1) S. A. Myslivets, ${ }^{1,2}$ V. A. Fokin, ${ }^{1}$ M. N. Volochaev, ${ }^{1}$ T. E. Smolyarova, ${ }^{1}$ \\ N. V. Radionov, ${ }^{1}$ A. I. Zaitsev, ${ }^{1}$ V. G. Arkhipkin, ${ }^{1,2}$ and A. S. Chirkin ${ }^{3}$ \\ ${ }^{1}$ Kirensky Institute of Physics, Federal Research Center KSC SB RAS, Krasnoyarsk 660036, Russia \\ ${ }^{2}$ Department of Photonics and Laser Technology, Siberian Federal University, Krasnoyarsk 660079, Russia \\ ${ }^{3}$ Faculty of Physics and International Laser Center, M. V. Lomonosov Moscow State University, Moscow 119992, Russia \\ *Corresponding author: vyunishev@iph.krasn.ru
}

Received 8 May 2019; revised 20 June 2019; accepted 1 July 2019; posted 1 July 2019 (Doc. ID 367032); published 24 July 2019

\begin{abstract}
A method for functional patterning of facets of a nonlinear crystal using focused ion beam milling has been developed. The near-field diffraction on periodic gratings has been experimentally and theoretically studied. The periodicity of the structure enables Talbot self-imaging at the fundamental and second-harmonic frequencies simultaneously. Spatial interference patterns for both harmonic frequencies are individual ones, which can enable the higher-accuracy optical testing, coupling the radiation at both frequencies, and wavelength-division demultiplexing. The impact of the aperture effect on a Talbot carpet is discussed. (c) 2019 Optical Society of America
\end{abstract}

https://doi.org/10.1364/OL.44.003761

When a plane wave is incident upon a periodic structure, the image of the structure is repeated at regular distances away from the structure plane. This phenomenon is known as the Talbot effect, or self-imaging, which leads to the diffraction self-reproduction of the image of a structure in the near field $[1,2]$. The distance $Z_{T}=2 \Lambda^{2} / \lambda$ (here $\Lambda$ is the grating period; $\lambda$ is the wavelength) between neighboring self-imaging planes is called the Talbot lengths. Images are also formed at the distance $z=(p / q) Z_{T}$, where $p$ and $q$ are coprime integers, but the image period is different from that of an object. This property is referred to as the fractional Talbot effect [3]. The Talbot effect can be used in photolithography [4,5], optical testing and metrology [6], imaging [7], spectrometry [8], etc. The recent progress in studying the Talbot effect and its applications were reviewed in Ref. [9]. Recently, the Talbot effect has been observed in nonlinear photonic crystals (NPCs) — a special kind of nonlinear media with the periodic bipolar modulation of the second-order nonlinear susceptibility [10] and the theory of the nonlinear Talbot effect have been developed [11].

At the same time, the nonlinear frequency conversion in periodic NPCs results in the nonlinear far-field RamanNath diffraction, which has been an object of many studies in the last decade [12-14]. In Ref. [15], the near- and far-field appearances of the nonlinear Raman-Nath diffraction in a periodically poled nonlinear crystal were simultaneously investigated. The main characteristic of the diffraction phenomena is the use of periodic structures that scatter radiation in certain directions. The simplest design of such a structure is a periodic phase grating, which has been intensively studied in many fields of research, except for the nonlinear frequency conversion. An exception is the use of patterned facets of nonlinear crystals for shaping both the fundamental and second-harmonic $(\mathrm{SH})$ beams in the far field, which was recently described in Ref. [16]. At the same time, the study of the near-field effects in facet patterned nonlinear crystals at dual harmonic frequencies is of interest in view of the close analogy between these effects in linear and nonlinear optics.

In this Letter, we investigate the Talbot effect using an optical grating on the exit facet of a nonlinear bulk medium. The incident radiation is scattered at the fundamental and doubled frequencies, so the Talbot self-imaging is reproduced at both harmonic frequencies at specified distances. The interference patterns for harmonic frequencies are individual ones, which can be used in the high-accuracy optical testing of patterned facets of nonlinear crystals and wavelength-division demultiplexing.

In the experiment, a strontium tetraborate $\left(\mathrm{SrB}_{4} \mathrm{O}_{7}, \mathrm{SBO}\right)$ crystal plate $1 \mathrm{~mm}(a) \times 3 \mathrm{~mm}(b) \times 5 \mathrm{~mm}(c)$ in size was used. Two periodic structures (gratings) with overall sizes of $100 \mu \mathrm{m} \times 100 \mu \mathrm{m}$ were formed on polished facet $a$ using a Hitachi FB-2100 focused ion beam system. The grating is a 1D structure of rectangular grooves parallel to the $c$ axis Fig. 1(a). The grating periods were designed to be 5 and $10 \mu \mathrm{m}$, respectively, and the groove depth was taken to be $1 \mu \mathrm{m}$. The fundamental frequency (FF) radiation at a central wavelength of $800 \mathrm{~nm}$ from a femtosecond oscillator delivering $100 \mathrm{fs}$ pulses at a repetition rate of $80 \mathrm{MHz}$ was focused by a $10 \mathrm{~cm}$ lens on a grating plane (facet $a$ ) placed on the exit facet of the sample. The fundamental radiation was polarized vertically (along the $c$ axis) to employ the largest SBO nonlinear coefficient $d_{33}$. The Raman-Nath diffraction at the FF and $\mathrm{SH}$ wavelengths was observed in the far field [Figs. 1(b) and $1(\mathrm{c})]$. One can see the first four diffraction orders of the fundamental beam and the $0, \pm 1, \pm 2, \pm 4$ orders of the SH beam. 
(a)
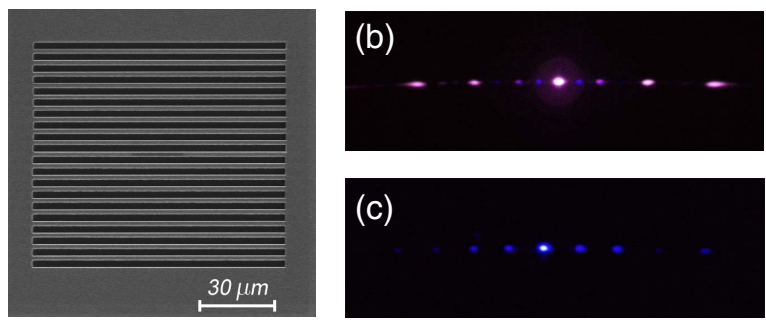

(d)

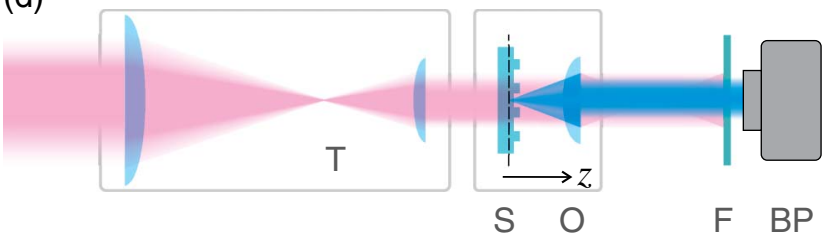

Fig. 1. (a) Focused ion beam image of the fabricated grating $(\Lambda=5 \mu \mathrm{m})$, (b) FF and $\mathrm{SH}$ far-field patterns $(\Lambda=10 \mu \mathrm{m})$, (c) the SH far-field pattern $(\Lambda=10 \mu \mathrm{m})$, and (d) the optical scheme of the Talbot effect experiment ( $T$, telescope; $S$, phase grating sample; $\mathrm{O}, 100 \times$ microscope objective mounted on the translation stage; optical filter F; BP, beam profiler).

An analysis of the angular positions of the FF and $\mathrm{SH}$ maxima in the far-field pattern revealed the discrepancy between the parameters of the fabricated structure and pre-designed ones. Therefore, the parameters were refined on a NanoInk atomic force microscope. The measured periods were 4.56 and $9.0 \mu \mathrm{m}$, which is consistent with the values obtained by analyzing the diffraction.

The measured depth of the grooves was $\sim 0.45 \mu \mathrm{m}$. The duty cycle of the examined grating $D$ with a period of $4.56 \mu \mathrm{m}$ was about 0.25 . Then the FF radiation was collimated by an optical telescope [Fig. 1(d)] to a slightly elliptic spot about $240 \mu \mathrm{m} \times 295 \mu \mathrm{m}$ in size (FWHM, xy plane) and launched upon the examined grating. A $100 \times$ microscope objective with a 1.25 numerical aperture was mounted on a translation stage to obtain the near-field FF intensity distribution in the transverse plane at a specified coordinate $z$. The intensity distribution was recorded by a Newport LBP-1 beam profiler placed at a distance of $180 \mathrm{~mm}$ from the sample. In the intensity distribution measurements at the $\mathrm{SH}$ wavelength, a BG39 glass filter was mounted before the beam profiler while, for the measurements of FF intensity distribution the BG39 glass filter was replaced by a neutral density filter of the laser beam profiler.

To analyze the spatial intensity distribution, the microscope objective was translated along the coordinate $z$. The scanning spatial step was $1.25 \mu \mathrm{m}$. At each step, the transverse intensity profiles were imaged by the optical scheme and beam profiler, and recorded by a data acquisition system. Figure 2 demonstrates sets of the FF and $\mathrm{SH}$ intensity profiles recorded at specified coordinates corresponding to the fractions of the corresponding Talbot lengths. The measured coordinates are given in Table 1 . It can be seen that the image at the distance $Z_{T}$ is a replica of the image corresponding to the output plane. Other Talbot effect features observed in our experiments are the halfperiod shift of the image at $1 / 2 Z_{T}$ image planes relative to the planes at integer values of $Z_{T}$ and double spatial frequency appearing at the $1 / 4 Z_{T}$ and $3 / 4 Z_{T}$ image planes. However, it is well established that the transverse intensity distribution at the
Table 1. Talbot Lengths for FF and SH Radiation Measured [from Figs. 3(a) and 3(c)], Calculated by Eq. (5), and Numerically Calculated [from Figs. 3(b) and 3(d)]

\begin{tabular}{lrrrrrrr}
\hline & \multicolumn{3}{c}{ FF $(\boldsymbol{\mu m})$} & & \multicolumn{3}{c}{ SH $(\boldsymbol{\mu m})$} \\
\cline { 2 - 3 } \cline { 7 - 8 }$Z / Z_{T}$ & Exp. & Eq. (5) & Num. & & Exp. & Eq. (5) & Num. \\
\hline $1 / 4$ & 12.50 & 13.00 & 12.75 & & 25.50 & 26.00 & 26.75 \\
$1 / 2$ & 25.00 & 26.00 & 25.75 & & 51.25 & 51.98 & 51.50 \\
$3 / 4$ & 37.50 & 39.00 & 37.50 & & 77.50 & 77.98 & 78.25 \\
1 & 50.00 & 51.98 & 51.75 & & 102.50 & 103.97 & 102.80 \\
$3 / 2$ & 73.75 & 77.98 & 76.75 & & 152.50 & 155.95 & 153.80 \\
2 & 98.75 & 103.97 & 102.50 & & 200.00 & 207.94 & 204.80 \\
\hline
\end{tabular}

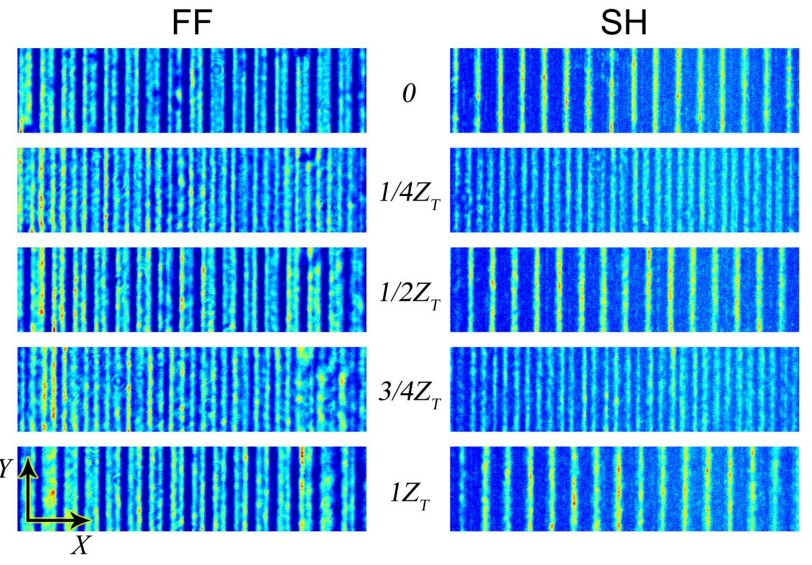

Fig. 2. Transverse FF (left column) and SH (right column) intensity distributions recorded by the beam profiler in the microscope objective positions of $0,1 / 4 Z_{T}, 1 / 2 Z_{T}, 3 / 4 Z_{T}$, and $Z_{T}$ positions at the corresponding wavelengths. The distances corresponding to the fractional planes are given in Table 1.

grating output plane and at the corresponding distance multiple of the Talbot length must be close uniformed for a pure phase grating [2]. Surprisingly, the intensity distributions at the output plane of the investigated structure were nonuniform at both frequencies (the upper row in Fig. 2). Therefore, the amplitude modulation appears at the output plane, which can be attributed to the light scattering by the rough groove surface and absorption of $\mathrm{Ga}$ ions unintentionally implemented during the ion milling procedure.

All the intensity profiles in the entire scanning range were averaged in the vertical direction (along the $c$ axis) to obtain the normalized intensity distribution in the transverse direction, and then aggregated to visualize the near-field intensity distribution in two dimensions ( $\{x, z\}$-coordinates), which is also known as a Talbot carpet. In Fig. 3(a), one can clearly see a periodic structure of the intensity distribution on the coordinates $\{x, z\}$. The periodicity of the structure in the $z$ direction was $50 \mu \mathrm{m}$ for FF radiation, which represents the Talbot length. In addition, it can be seen that the diffraction caused by a finite grating size disturbs the regularity of this spatial distribution. Similar results were obtained for the $\mathrm{SH}$ radiation [Fig. 3(c)]; the measured Talbot length for the $\mathrm{SH}$ radiation was found to be $102.5 \mu \mathrm{m}$, which is about twice as much as the Talbot length at the FF wavelength. 
(a)
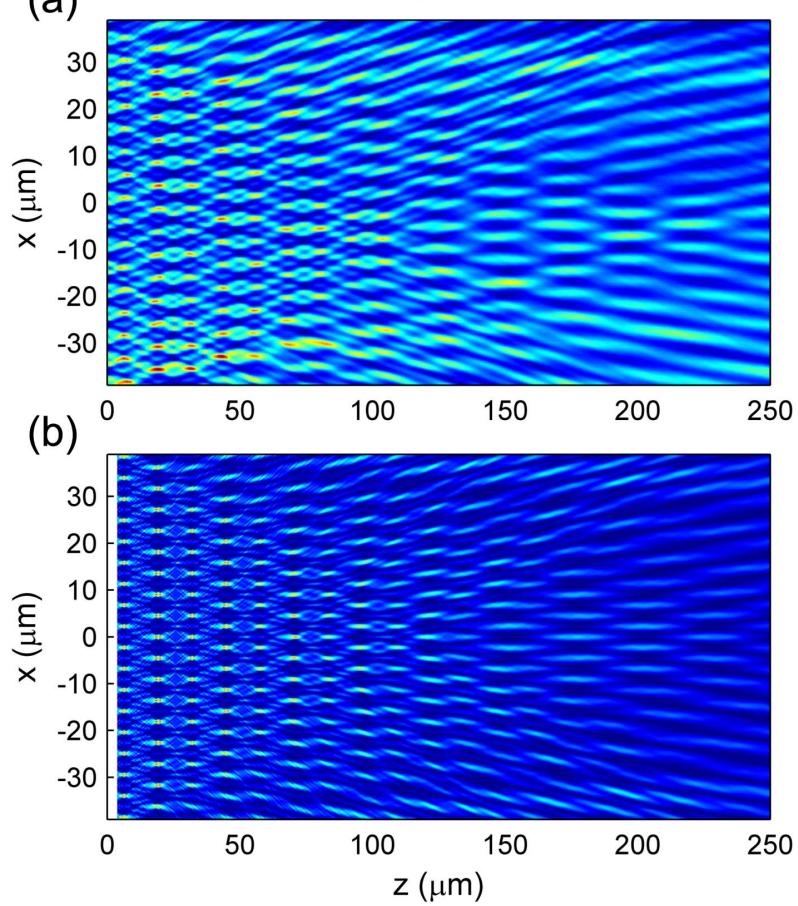

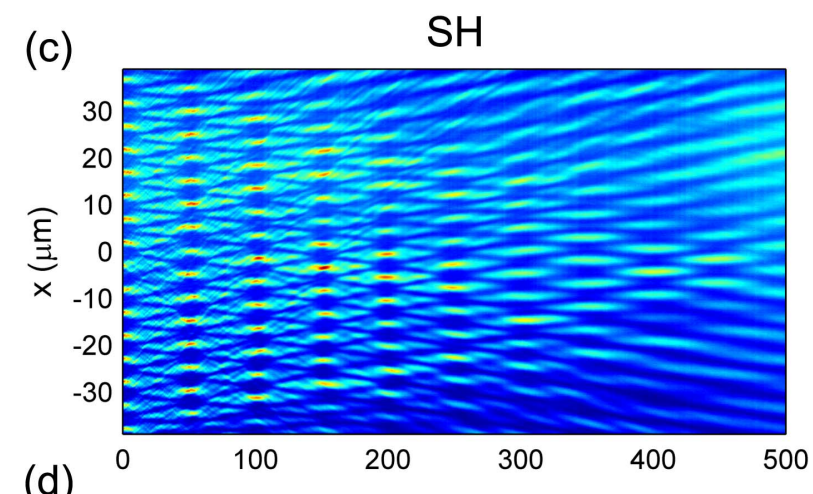

(d)

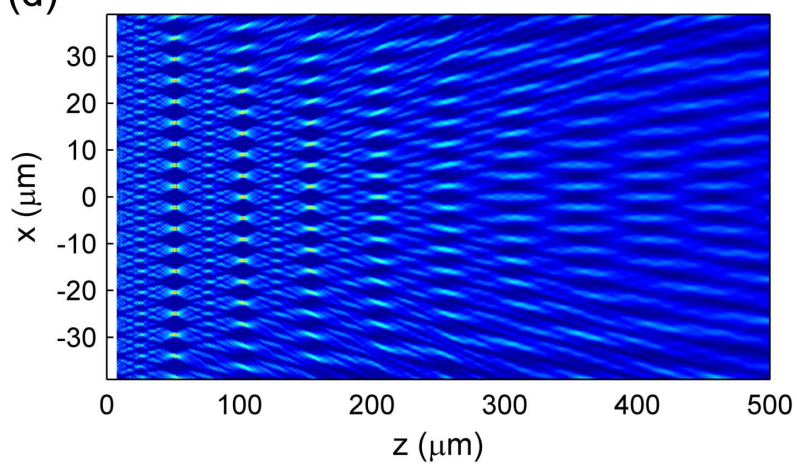

Fig. 3. (a), (c) Measured; (b), (d) calculated using Eq. (1) (a), (b) spatial FF and (c), (d) SH intensity distributions in the near field.

A simple theoretical treatment might rely on the Green function formalism $[17,18]$. In this case, the complex field amplitude in the quasi-static approximation can be written as

$$
A(\mathbf{r}, z)=\int_{S} g(\boldsymbol{\rho}) A^{(0)}(\rho) G(\mathbf{r}-\boldsymbol{\rho}, z) \mathrm{d} \boldsymbol{\rho} .
$$

Here $G(\mathbf{r}-\boldsymbol{\rho}, z)$ is the Green's function of the parabolic equation

$$
G(\mathbf{r}-\boldsymbol{\rho}, z)=-\frac{i k}{2 \pi z} e^{-i \frac{k}{2 z}(\mathbf{r}-\boldsymbol{\rho})^{2}},
$$

$k=2 \pi / \lambda$ is the wavevector of the FF or SH wave, $\mathbf{r} \in(X, Y)$ is the coordinate in the image plane, $\boldsymbol{\rho} \in(x, y)$ is the coordinate in the object plane, $z$ is the propagation coordinate, $g(\boldsymbol{\rho})$ is the periodic modulation function, $A^{(0)}(\boldsymbol{\rho})=A(0) \exp \left(-\boldsymbol{\rho}^{2} / w_{0}^{2}\right)$, $A(0)$ is the amplitude in the beam axis, $w_{0}$ is the beam radius, and $S$ is the integration area.

The surface grating results in the amplitude-phase modulation at the crystal output plane, such that the periodic modulation function $g(\boldsymbol{\rho})$ can be represented as

$$
g(x)=\left\{\begin{array}{ll}
e^{-i \phi_{1,2},} & \text { if } p \Lambda \leq x<p \Lambda+d \\
\xi_{1,2}, & \text { if } p \Lambda+d \leq x<(p+1) \Lambda
\end{array},\right.
$$

where $p$ is the period number (e.g., $p=1$ ), and $\phi_{1,2}=$ $2 \pi\left(n_{1,2}-1\right) h \lambda_{1,2}$ is the phase increment caused by the groove depth $h, n_{1,2}$ are the refractive indexes at the wavelengths $\lambda_{1,2}$, $d=D \Lambda$ is the groove thickness, $\xi_{1,2}$ are the transmission $\left(\xi_{1,2} \in[0,1]\right)$. The surface phase gratings are defined by a set of independent variable parameters (period, duty cycle, groove depth, etc.), in contrast to the two parameters (period and duty cycle) in a conventional NPC. This means that by varying the groove depth, one can set a phase modulation magnitude during fabrication.
It can be shown that the Talbot plane of the $m$ th order under focusing is

$$
Z_{T}^{(m)}=\frac{m Z_{T}^{(0)}}{2 \gamma^{2}}\left[1-\left(1-4 \gamma^{2}\right)^{1 / 2}\right],
$$

where $\gamma=m Z_{T}^{(0)} / Z_{R}=m \Lambda^{2} / \pi w_{0}^{2}, Z_{T}^{(0)}=2 \Lambda^{2} / \lambda$ is the Talbot length in the plane wave approximation [9], $\Lambda$ is the grating period, $\lambda$ is the wavelength of the incident plane wave, and $Z_{R}=\pi w_{0}^{2} / \lambda$ is the diffraction length (also known as a Rayleigh length). According to Eq. (4), we have $Z_{R}>2 Z_{T}^{(0)}$ or $w_{0}>\Lambda$. Thus, the Talbot effect can occur, even though the beam illuminates few grating periods. Making the assumption $4 \gamma^{2} \ll 1$ in Eq. (4), we can simplify the latter to

$$
Z_{T}^{(m)}=m Z_{T}^{(0)}\left[1+\left(\frac{m \Lambda^{2}}{\pi w_{0}^{2}}\right)^{2}\right]
$$

Equation (5) ensures the high accuracy under the experimental conditions and can be used instead of Eq. (4). According to Eq. (5), the Talbot lengths at both frequencies are interrelated as $2 Z_{T}(\lambda) \approx Z_{T}(\lambda / 2)$. Therefore, the effect provided by the facet patterned nonlinear crystals can be used for coupling the radiation at both frequencies to multi-channel integrated optical circuits. On the other hand, the self-images at both frequencies are laterally shifted by half a period at the coordinates $Z_{T}(\lambda) \approx 1 / 2 Z_{T}(\lambda / 2)$, allowing the spatial separation of harmonic waves. The values for the entire and fractional Talbot lengths measured and calculated using Eq. (5) are given in Table 1. These measured and calculated data were found to be in satisfactory agreement.

Figure 3(b) demonstrates the near-field intensity distribution for the FF radiation obtained by the numerical integration of Eq. (1) in a finite domain. In the calculations, the quasi-static 
and paraxial approximations were used, and the propagation of a Gaussian beam at a single FF wavelength was considered. It is worth noting that the pulse duration does not influence the Talbot effect under the condition $\tau \gg t$ (here $\tau$ is the pulse duration, $t \sim h / c$, and $c$ is the speed of light), and Eq. (1) is valid. The spatial increment in the calculations was taken to be $0.25 \mu \mathrm{m}$. The calculated and measured intensity distributions agree well at the following grating structure parameters: $\Lambda=4.53 \mu \mathrm{m}, D=0.25$, a groove depth $h=0.52 \mu \mathrm{m}$, and a transmission of $\xi_{1}=0.8$. The groove depth affects the relative phases $\phi_{1,2}$ for the radiation, while the necessity for introducing the amplitude $\xi_{1}$ can be explained by the light scattering by the rough groove surface. In contrast to the results obtained at the FF wavelength, the measured spatial $\mathrm{SH}$ intensity distribution contains noticeable peaks at the object plane, which are indicative of the amplitude modulation at the grating (Fig. 2). For this reason, the amplitude $\xi_{2}$ in Eq. (3) was varied. As a result, good agreement between the calculated and measured Talbot carpets is also observed at $\xi_{2}=0.2$ [Figs. 3(b) and 3(d)]. As was mentioned above, it may appear due to the light scattering by the rough groove surface and absorption of gallium ions in the short-wavelength range. The latter factor seems to dominate for the $\mathrm{SH}$ radiation. The optimization of the grating parameters allowed us to obtain better agreement between the numerically calculated and measured Talbot lengths (in Table 1), as compared with the values obtained using Eq. (4). Hence, the Talbot self-imaging at the FF and $\mathrm{SH}$ frequencies can be used in the more accurate optical testing of periodically patterned facets of nonlinear crystals.

The Talbot effect is usually observed when the grating is illuminated by a monochromatic (continuous) wave. In our experiments, $100 \mathrm{fs}$ pulses were used, and no discrepancy between the numerically calculated and measured data was observed, which evidences for the validity of the monochromatic approximation. This is consistent with the data reported in Ref. [19], where the Talbot effect was shown to occur under the short-pulse illumination at a pulse duration down to $30 \mathrm{fs}$.

Although the spatial period of the radiation at the $\mathrm{SH}$ frequency is twice as much as at the FF frequency, only two Talbot planes are observed in both cases. Then the self-images experience certain distortions. This is proved to be caused mainly by a finite area of the structure, i.e., a small number of the periods illuminated by the FF radiation.

In summary, we studied the Talbot self-imaging at the harmonic frequencies using facet patterned nonlinear crystals structured by focused ion beam milling. The near-field diffraction at the periodic gratings was experimentally and theoretically investigated. The periodicity of the structure enables the Talbot self-imaging at the fundamental and secondharmonic frequencies simultaneously. Spatial interference patterns for both harmonic frequencies are individual ones due to the different phase increment and the presence of the spatial amplitude modulation in the case of SH. This opens new possibilities for the high-accuracy optical testing, coupling the radiation at both frequencies to multichannel optical circuits, and wavelength-division demultiplexing. The influence of the aperture effect on the Talbot carpet was discussed.

Funding. Russian Science Foundation (RSF) $(19-$ 12-00203).

Acknowledgment. The surface gratings were fabricated in the Center of Collective use of FRC KSC SB RAS.

\section{REFERENCES}

1. H. F. Talbot, Philos. Mag. 9, 401 (1836).

2. K. Patorski, The Self-Imaging Phenomenon and Its Applications, Vol. 27 of Progress in Optics (Elsevier, 1989), Chap. 1, pp. 1.

3. M. V. Berry and S. Klein, J. Mod. Opt. 43, 2139 (1996).

4. L. Stuerzebecher, T. Harzendorf, U. Vogler, U. D. Zeitner, and R. Voelkel, Opt. Express 18, 19485 (2010).

5. H. S. Kim, W. Li, S. Danylyuk, W. S. Brocklesby, M. C. Marconi, and L. Juschkin, Opt. Lett. 39, 6969 (2014).

6. D. S. Mehta, S. K. Dubey, C. Shakher, and M. Takeda, Appl. Opt. 45, 7602 (2006)

7. S. Feng, M. Wang, and J. Wu, Opt. Lett. 41, 3157 (2016).

8. E. Ye, A. H. Atabaki, N. Han, and R. J. Ram, Opt. Lett. 41, 2434 (2016).

9. J. Wen, Y. Zhang, and M. Xiao, Adv. Opt. Photonics 5, 83 (2013).

10. Y. Zhang, J. Wen, S. N. Zhu, and M. Xiao, Phys. Rev. Lett. 104 183901 (2010).

11. J. Wen, Y. Zhang, S.-N. Zhu, and M. Xiao, J. Opt. Soc. Am. B 28, 275 (2011).

12. S. M. Saltiel, D. N. Neshev, W. Krolikowski, A. Arie, O. Bang, and Y. S. Kivshar, Opt. Lett. 34, 848 (2009).

13. S. M. Saltiel, D. N. Neshev, W. Krolikowski, N. Voloch-Bloch, A. Arie, O. Bang, and Y. S. Kivshar, Phys. Rev. Lett. 104, 083902 (2010).

14. A. M. Vyunishev, V. V. Slabko, I. S. Baturin, A. R. Akhmatkhanov, and V. Y. Shur, Opt. Lett. 39, 4231 (2014).

15. D. Liu, D. Wei, M. Gu, Y. Zhang, X. Hu, M. Xiao, and P. Han, Opt. Lett. 43, 3168 (2018).

16. D. Singh, R. Shiloh, and A. Arie, Opt. Mater. Express 8, 2654 (2018).

17. J. Goodman, Introduction to Fourier Optics, McGraw-Hill Physical and Quantum Electronics Series (W. H. Freeman, 2005).

18. S. Akhmanov and S. Nikitin, Physical Optics (Clarendon Press, 1997).

19. H. Wang, C. Zhou, S. Zhao, P. Xi, and L. Liu, J. Opt. A 3, 159 (2001). 